I have also found the modified expression to be applicable to hydride di-atoms in the period from $\mathrm{LiH}$ to FH. Morse's function, far from remaining constant, is definitely periodic in the earlier periods of both hydride and non-hydride di-atoms.

C. H. Douglas Clark.

Department of Inorganic Chemistry, University, Leeds, 2. June 20.

C. H. Douglas Clark, NaTURE, 133, 873, June 9, 1934.

2 W. L. Bragg, Phil. Mag., vi, 40, $169 ; 1920$.

"X. V. Sidgwick "The Covalent Link in Chemistry" (Cornell University Press, 1933) (see Table XVIII on p. 85).

. (Cambridge University Press, 1932) (see Appendix II, p. 280).

\section{$L$ Absorption Spectra in the Very Soft X-Ray Region}

THE $L_{\mathrm{III}}$ absorption edges of aluminium and magnesium have been measured. Since the continuous X-ray spectrum in this region (above $100 \mathrm{~A}$.) is very feeble, I have used optical spark spectra from elements giving spectra rich in lines. As source for the spectra reproduced in Fig. 1 a spark between copper electrodes was used. The apparatus was a concave grating spectrograph with a glass grating, ruled at this laboratory, of radius $1 \mathrm{~m}$., 288 lines per mm.

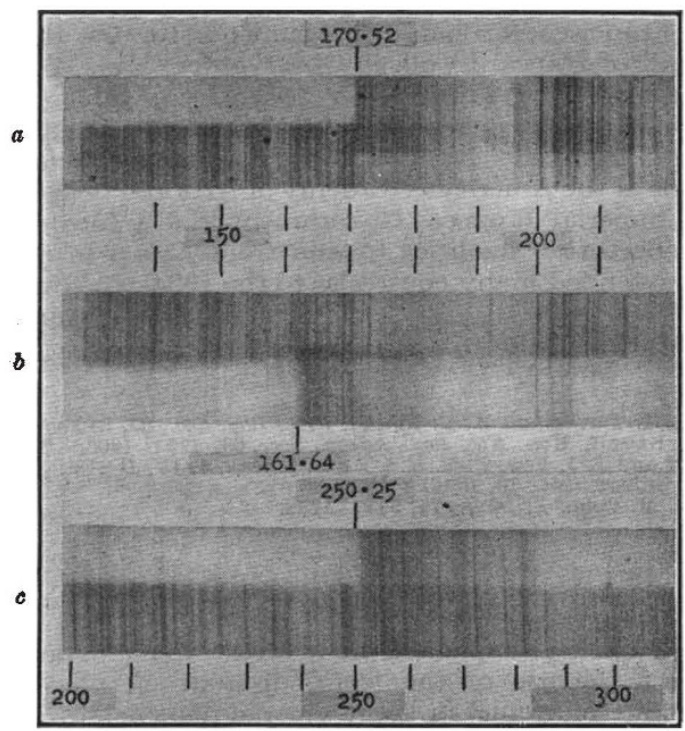

Fra. 1. $L_{\mathrm{III}}$ absorption limits of (a) $\mathrm{Al},(b) \mathrm{Al}$ in $\mathrm{Al}_{2} \mathrm{O}_{3}$, and $(c) \mathrm{Mg}$.

The thickness of the foils used was for aluminium $0.5 \mu$ and for magnesium about $0.3 \mu$. The magnesium was deposited on a $0 \cdot 5 \mu$ aluminium foil. The wavelengths for the copper lines in this region being known ${ }^{1}$, the edges were measured relative to those lines. The $L_{\mathrm{III}}$ absorption limit of $\mathrm{Al}$ was found to be $170 \cdot 52 \mathrm{~A}$., giving $\nu / R=5 \cdot 344$. The corresponding value for $\mathrm{Mg}$ was $250.25 \mathrm{~A}$., $\nu / R=3.641$.

I have also measured the $L_{\mathrm{III}}$ absorption edge of $\mathrm{Al}$ in $\mathrm{Al}_{2} \mathrm{O}_{3}$ (Fig. 1, b). The value found is $161 \cdot 64 \mathrm{~A}$.

A detailed report will be given elsewhere.

V. Hugo SANnER.

Physics Laboratory,

University, Upsala.

May 29.

${ }^{1}$ Kruger and Cooper, Phys. Rev., 44, 826; 1933.

\section{Heat Flow during Surface Colour Formation}

When gaseous substances slowly react with metals, and the volume of the solid resultants is greater than that of the metal at the temperature of reaction, a series of bright interference colours is generally produced. Some anomalous features are shown, however, when the dynamies of the formation of the films are considered, and occur generally independently of the gaseous reagent and the nature of the solid metal. The rate of formation slows down faster than described by the laws of diffusion through a thickening film at constant temperature, and, further, if the gaseous reactant be removed, causing the interruption of the reaction, the speed at which the reaction re-occurs diminishes. The longer the time of arrest the more marked is the slowing down, and in some cases, for example, temper colours on steel, the colour appears to be fixed.

The molecular heat evolution in this type of reaction is large, but the films are so thin that ordinary methods fail to detect the heating, and in reactions at room temperature the film is cold if touched. While the reaction is proceeding, the film is separated from the metal by an interface at which the heat evolution is proceeding, and a partition of the heat evolved occurs, the metal taking most by reason of its large thermometric conductivity. On account of the relatively enormous mass of the metal, it appears little altered in temperature. The interference film is thin, and the heat remaining in it, though much less than that flowing into the metal, produces a marked elevation of the temperature while the film is forming.

The order of the effect for iron, nickel and copper, when one oxide colour sequence is produced in about two minutes, is $20^{\circ} \mathrm{C}$. for copper oxide, $50^{\circ} \mathrm{C}$. for nickel oxide, and $90^{\circ} \mathrm{C}$. for an iron oxide film. Thus the diffusion of the reactants through the oxide film is cut down by a purely physical cause if the reaction be arrested, since the film can then attain the temperature of the mass of metal relatively quickly. In each case after interruption the same velocity can only be attained again by raising the temperature, and the rise in temperature necessary after a considerable interruption is of the same order as the original elevation of the temperature of the film before the reaction was stopped.

In the same way, the dynamics of the slow attack of gaseous reagents on metals is complicated by the initial higher temperature of the interference film falling when the rate of evolution of heat at the interface becomes comparable with the rate of flow of the heat stored in the interference film through the massive metal. The fall of the velocity under these conditions is thus autocatalytic, and affords a ready explanation of the fact that sometimes experience leads to the association of a definite colour of the final film with a definite temperature for the plane surfaces of massive metal. The greater the molecular heat of reaction, and the smaller the difference between the thermometric conductivity of the metal and the interference film, the more noticeable becomes the fixed colour at a fixed temperature in the range of temperatures in which the attack is slow.

\section{F. H. Constable.}

St. John's,

Cashio Lane, Letchworth. 